\title{
Evaluation of Vitamin B12 status in type 2 Diabetes Mellitus Patients on Metformin therapy
}

\author{
Ananda Vayaravel Cassinadane ${ }^{1}$, Ramesh Ramaswamy², Shaik Anwar Hussain ${ }^{3}$, \\ Radhika Govindarajan ${ }^{4}$, Abu Raghavan Srinivasan ${ }^{5}$ \\ ${ }^{1}$ Tutor cum PhD scholar, Department of Biochemistry, Sri Venkateshwaraa Medical College Hospital and Research \\ Centre (SVMCHRC), Ariyur, Puducherry, ${ }^{2}$ Professor and Head, Department of Biochemistry, JIPMER, Karaikal, \\ Puducherry, ${ }^{3}$ Associate Professor, Department of Medicine, Sri Venkateshwaraa Medical College Hospital \\ and Research Centre (SVMCHRC), Ariyur, Puducherry, ${ }^{4}$ Professor and Head, Department of Biochemistry, Sri \\ Venkateshwaraa Medical College Hospital and Research Centre (SVMCHRC), Ariyur, Puducherry, ${ }^{5}$ Professor, \\ Department of Biochemistry, Mahatma Gandhi Medical College and Research Institute (SBV) University, Puducherry
}

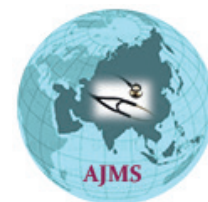

A B S TR A C T

Background: B12 deficiency is a silent epidemic with serious consequences. Long term use of metformin has been associated with malabsorption of vitamin B12 leading to its deficiency along with increased homocysteine levels. With this background we designed this study to assess the status of vitamin B12 and its markers in type 2 diabetes mellitus patients on metformin therapy who are attending tertiary care hospital in the peripheral area of Pondicherry. Aims and Objective: The current study aims to study the relationship if any, between the glycemic status and the status of Vitamin B12 among T2DM patients on metformin therapy. Materials and Methods: A case control study was done with a sample size of 100 (50 case and 50 control). The patients were selected according to the inclusion criteria and their blood sample were analyzed for the various parameters. The markers of vitamin B12 status are studied among both the control and the patients with T2DM on metformin therapy and compared with their glycaemic status. Results: There is a significant increase in the levels of FBS, HbA1c, insulin and C-peptide in the study group. It was observed that the mean levels of homocysteine and Methylmalonic acid were significantly higher with lower levels of vitamin B12 in patients who were on metformin therapy. Conclusion: There is moderate correlation between the markers of B12 status and levels of fasting blood sugar as well as $\mathrm{HbA} 1 \mathrm{C}$. There is a significant correlation between insulin and C-peptide with the markers of vitamin B12 status.

Key words: B12 deficiency markers, Glycemic status, Metformin, Vitamin B12 deficiency, Type 2 diabetes mellitus

\section{INTRODUCTION}

One of the efficient anti-diabetic drug forming a milestone in the treatment of T2DM is Metformin. This biguanide is prescribed clinically for the past 60 years as a first line oral anti-diabetic agent. Every year Metformin caters to the need of more than 150 million people, the reason being its therapeutic efficacy and affordable price. ${ }^{1}$ The mechanism of action of Metformin in improving hyperglycemia involves in improving the signaling of insulin together with suppressing hepatic gluconeogenesis.
Vitamin B12 deficiency results as one of the few side effects of metformin usage. This is being overlooked and rarely investigated owing to the numerous clinical benefits of metformin. ${ }^{2}$ Even well-educated health providers overlook such an easily diagnosed and inexpensively treated condition which leads to a frustrating B12 deficiency epidemic. Untreated B12 deficiency can cause hypercellular and dysplastic bone marrow. This can be mistaken for signs of acute leukaemia.It is to be noted here that B12 levels are normal $50 \%$ of the patients with subclinical disease. ${ }^{3}$ 
Vitamin B12 plays a vital role in DNA and cellular metabolism by serving as an essential cofactor in methylation process. Hence a deficiency leads to DNA disruption and derangement of cellular metabolism. This might lead to serious clinical consequences. Vitamin B12 is intracellularly converted to twoactive coenzymes, adenosylcobalamin in mitochondria and methylcobalamin in the cytoplasm. They are necessary for the homeostasis of Methyl malonic acid (MMA) and Homocysteine (HC). Methylmalonic acid is converted intosuccinyl-CoA, of which vitamin B12 is a cofactor for the reaction. Homocysteine is biosynthesized from methionine then resynthesized into methionine or converted into amino acidcysteine. ${ }^{4}$

The markers which are increased early in vitamin B12 deficiency include serum methyl malonic acid and homocysteine.Measurement of serum methyl malonic acid and homocysteine levels are more sensitive methods of screening for vitamin B12 deficiency. ${ }^{5}$ High levels of methyl malonic acid and homocysteine have been identified as better indicators of B-12 deficiency than the actual serum B-12 level itself.A deficiency of vitamin B-12at the tissue level elevates the levels of both MMA and HC even when serum vitamin B-12 concentrations are within the reference values. ${ }^{5}$ Elevated MMA and HC levels together havebeen found to be $99.8 \%$ sensitive for diagnosing functional vitamin B-12 deficiency. ${ }^{6}$

\section{MATERIALS \& METHODS}

100 blood samples ( 50 controls +50 cases) were collected from SriVenkateshwaraa Medical College Hospital and Research Centre (SVMCHRC). Cases included the T2DM patients on metformin therapy, who attended the clinic of SVMCHRC, Ariyur, Pondicherry. The patients were selected as per the following criteria.

\section{Inclusion criteria}

1. T2 DM patients of the age group 35-55 of both sex who were on Metformin

2. Duration of treatment for a minimum of 6 months

3. Dosage of metformin of at least $1000 \mathrm{mg} /$ day.

\section{Exclusion criteria}

1. Type 2 Diabetic Patients with any co existing cause for B12 deficiency,

2. Pregnant ladiesandType 2 Diabetic Patients on other drugs.

3. Malabsorption syndromes.

Samples were analyzed for the following study variables vitamin B12, homocysteine, methyl malonic acid, plasma insulin and C-Peptide using chemiluminescence (ROCHE Cobas e 411).HbA1C was estimated by HPLC method(Biorad D10).
Statistical analysis was done by SPSS 17 version. Shapiro-Wilk test was performed to see whether the continuous variables follow normal Gaussian distribution. To compare vitamin B12 and glycemic status, student ' $\mathrm{t}$ ' test was performed and to find the correlation between vitamin B12 deficiency markers with glycemic status in cases, Pearson correlation analysis was performed. P value less than 0.05 was considered as significant.

\section{RESULTS}

Total of 100 subjects were included in the study based upon the inclusion and exclusion criteria, after obtaining the informed consent. 100 subjects were divided into 2 groups, Case (T2DM patients on metformin therapy) and control (normal healthy subjects). Final statistical analysis were performed and the results were depicted in the tabular column.

Results showed significant increased levels of FBS, $\mathrm{HbA1c}$,insulin and C-peptide in the study group. It was observed that the mean levels of vitamin B12 markers homocysteine and methylmalonic acid were significantly higher and low level of vitamin B12 was found in patients who were on metformin therapy (Table 1).

We have correlated the markers of glycemic status with the markers of Vitamin B12 deficiency Homocysteine and Methylmalonic acid. The results showed positive correlation between the two markers was depicted in Table 2 and Figure $1(\mathrm{a}, \mathrm{b}, \mathrm{c}, \mathrm{d})$ and Figure $2(\mathrm{a}, \mathrm{b}, \mathrm{c}, \mathrm{d})$ respectively.

\begin{tabular}{lccc} 
Table 1: Characteristics of glycemic and vitamin \\
b12 status compared with normal and T2DM on \\
metformin \\
Biochemical \\
markers & Control (150) & Case (150) & P value \\
\hline Fasting glucose & $87.4 \pm 18.7$ & $153 \pm 75$ & 0.0001 \\
Fasting insulin & $6.18 \pm 3.03$ & $8.28 \pm 4.18$ & 0.0001 \\
HbA1c\% & $4.9 \pm 0.6$ & $9.6 \pm 3.3$ & 0.0001 \\
C-peptide & $2.3 \pm 0.9$ & $4.1 \pm 0.4$ & 0.0001 \\
Vitamin B12 & $372.38 \pm 67.9$ & $215.92 \pm 66.44$ & 0.0001 \\
Homocysteine & $6.17 \pm 2.87$ & $12.3 \pm 4.5$ & 0.0001 \\
Methyl Malonic & $122 \pm 82.5$ & $154.5 \pm 0.84 .5$ & 0.0001 \\
acid & & & \\
\hline
\end{tabular}

\begin{tabular}{|c|c|c|}
\hline $\begin{array}{l}\text { Parameters } \\
\text { (T2 DM patients) }\end{array}$ & MMA (r) & Homocysteine(r) \\
\hline Insulin & 0.75 & 0.78 \\
\hline C-peptide & 0.78 & 0.90 \\
\hline $\mathrm{HbA} 1 \mathrm{c}$ & 0.6 & 0.58 \\
\hline FBS & 0.49 & 0.52 \\
\hline
\end{tabular}

Asian Journal of Medical Sciences | Mar-Apr 2018 | Vol 9 | Issue 2 

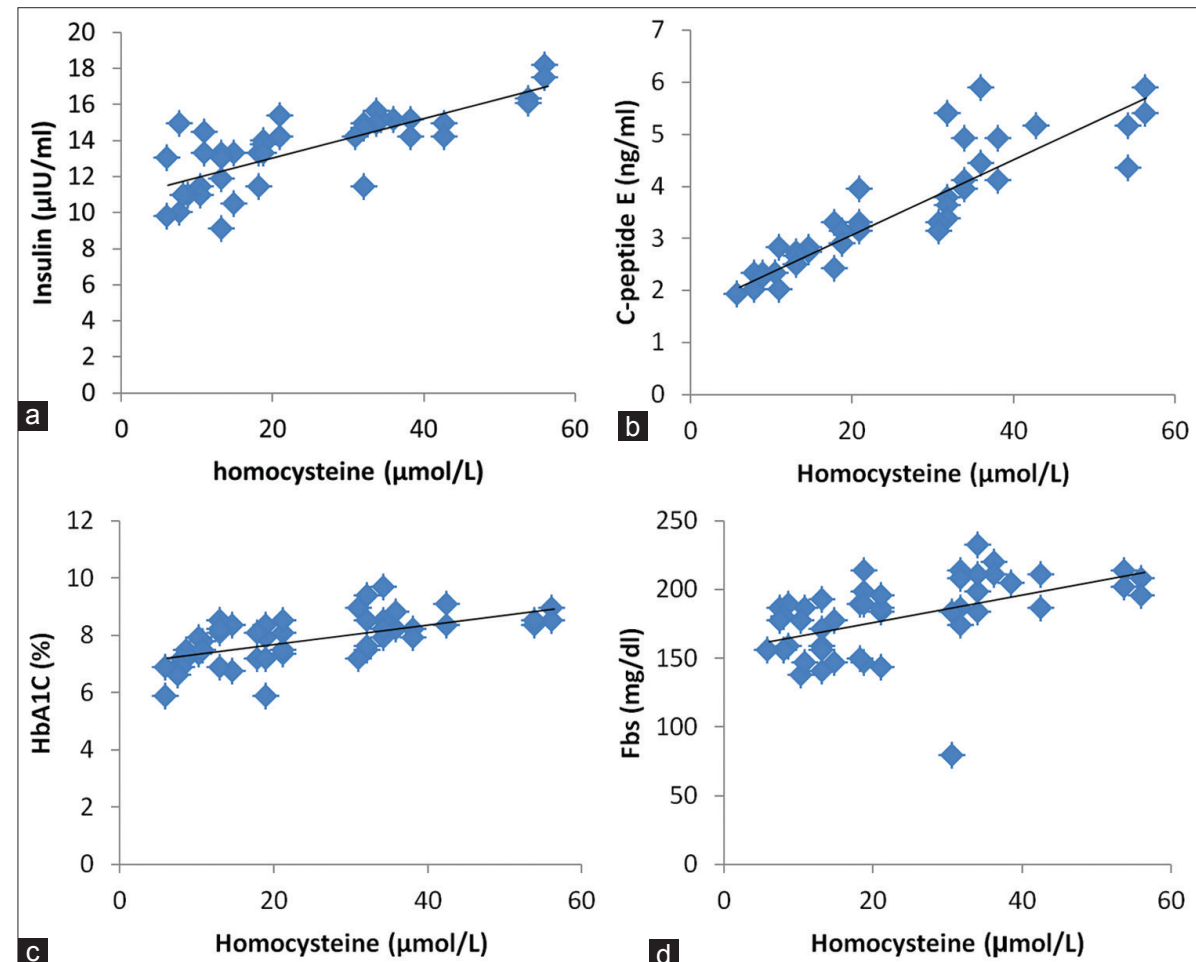

Figure 1: Homocysteine versus glycemic status. (a) Homocysteine Vs Insulin, (b) Homocysteine Vs C-peptide, (c) Homocysteine Vs HbA1C, (d) Homocysteine Vs Fasting blood sugar
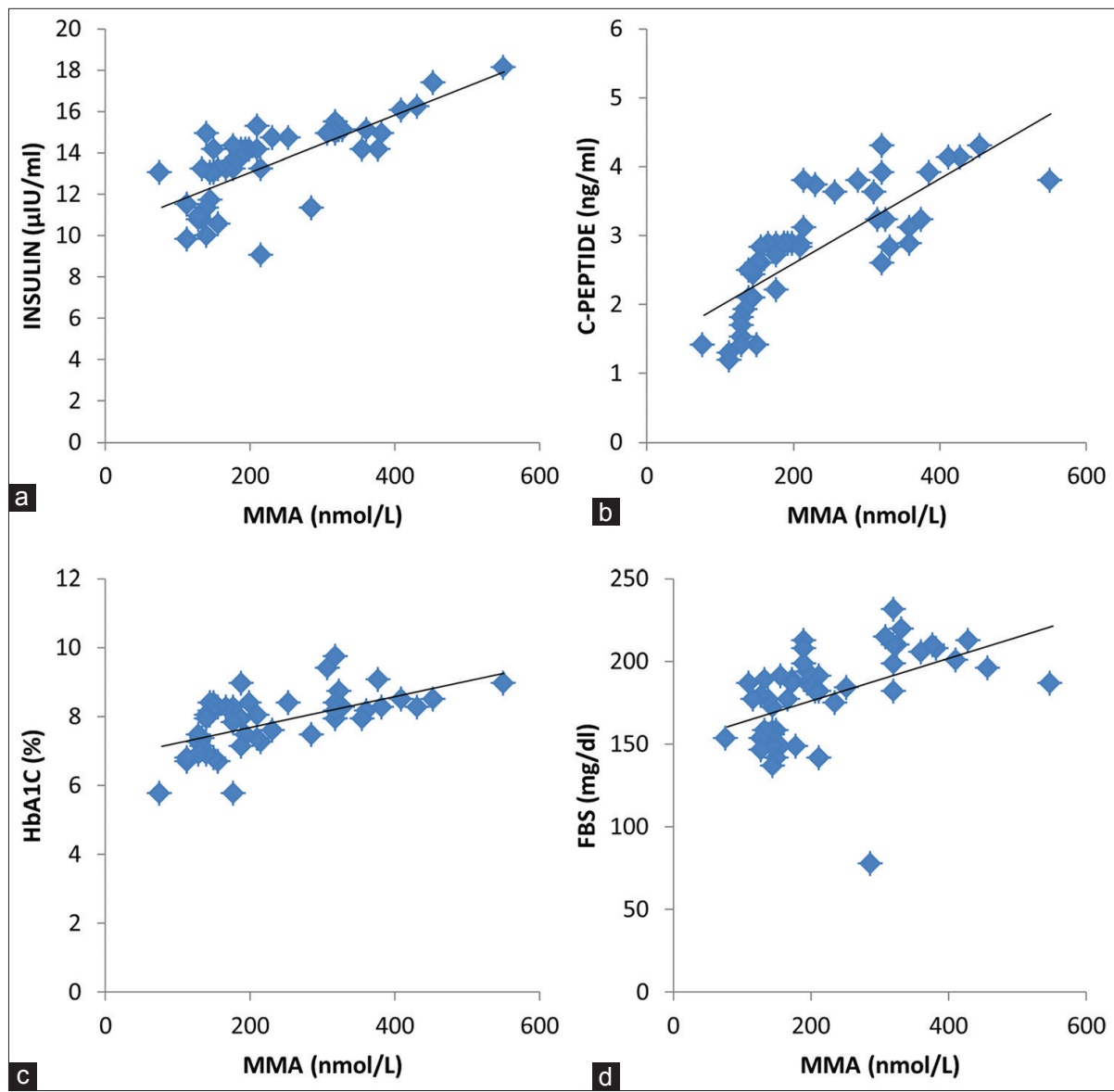

Figure 2: Methylmalonic acid versus glycemic status, (a) Methylmalonic acid Vs insulin, (b) Methylmalonic acid Vs C-peptide, (c) Methylmalonic acid Vs HbA1c, (d) Methylmalonic acid Vs Fasting blood sugar 


\section{DISCUSSION}

Total serum vitamin B12 is a relatively insensitive and unspecific biomarker of deficiency that does not reflect recent variations in cobalamin status. Holotranscobalamin (holoTC), the metabolically active portion of vitamin B12, is the earliest laboratory parameter that becomes decreased in case of a vitamin B12 negative balance. Concentration of methylmalonic acid (MMA) is a functional vitamin B12 marker that will increase when the vitamin B12 stores are depleted. Isolated lowering of holo'TC shows vitamin B12 depletion (negative balance), while lowered holoTC plus elevated MMA (and homocysteine) indicates a metabolically manifested vitamin B12 deficiency, although there still may be no clinical symptoms. ${ }^{7}$

The concentration of total homocysteine (tHcy) in serum and plasma is elevated in both folate and cobalamin deficiencies, whereas methylmalonic acid (MMA) in serum, plasma, or urine is a specific marker of cobalamin function. The combined measurement of both metabolites is useful for the diagnosis and follow-up of these deficiency states. In addition, total homocysteine is elevated under various pathologic states (eg, renal failure), and hyperhomocysteinemia is associated with an increased risk of cardiovascular disease, cognitive dysfunction, and adverse pregnancy outcomes. ${ }^{8}$

The relationship between insulin and homocysteine is controversial. However, handful of studies have found strong associations between insulin and homocysteine. One study in humans found that B-12 and Folate therapy resulted in a number of improved parameters among patients with metabolic syndrome. ${ }^{9}$

$\mathrm{HbA1c}$ is an independent risk factor for type 2 diabetes. Subjects with high-normal levels of $\mathrm{HbA1c}$ deserve particular attention because they have a strong risk of developing diabetes. ${ }^{10}$ In this study the correlation between fasting blood glucose and $\mathrm{HbA1c}$ was analyzed with the markers of vitamin B12 and no correlation existed between them.

In the transition from normal glucose tolerance (NGT) to type 2 diabetes mellitus (T2DM), the role of $\beta$-cell dysfunction and peripheral insulin resistance (IR) is well established.In this study there is a significant correlation between insulin and C-peptide with the markers of vitamin B12 status.

\section{CONCLUSION}

There is moderate correlation between the markers of B12 status and levels of fasting blood sugar as well as HbA1C. There is a significant correlation between insulin and C-peptide with the markers of vitamin B12 status.

\section{ACKNOWLEDGEMENT}

Faculties of the department of Biochemistry, SVMCHRC, Pondicherry. Research scholars Mr. V. Kuzhandai Velu and Mr. M. Lenin, department of Biochemistry, Mahatma Gandhi Medical College and Research Institute (SBV), Pondicherry.

\section{REFERENCES}

1. An $\mathrm{H}$ and $\mathrm{He} \mathrm{L}$. Current understanding of metformin effect on the control of hyperglycemia in diabetes. J Endocrinol 2016; 228: R97-R106.

2. Akinlade KS, Agbebaku SO, Rahamon SK and Balogun WO. Vitamin B12 levels in patients with type 2 Diabetes Mellitus on Metformin. Ann Ib Postgrad Med 2015; 13: 79-83.

3. Pacholok SM. Vitamin B12 Deficiency: Serious Consequences. Available at: http://www.pharmacytimes.com/publications/ issue/2013/december2013/vitamin-b12-deficiency-seriousconsequences. (Accessed: $25^{\text {th }}$ December 2017)

4. Hunt A, Harrington D and Robinson S. Vitamin B12 deficiency. BMJ 2014; 349: g5226-g5226.

5. Oh R and Brown DL. Vitamin B12 deficiency. Am Fam Physician 2003; 67: 979-986.

6. Savage DG, Lindenbaum J, Stabler SP and Allen RH. Sensitivity of serum methylmalonic acid and total homocysteine determinations for diagnosing cobalamin and folate deficiencies. Am J Med 1994; 96: 239-246.

7. Herrmann W and Obeid R. Holotranscobalamin - An Early Marker for Laboratory Diagnosis of Vitamin B12 Deficiency. Eur Oncol Haematol 2009;03:7.

8. Monsen AL, RefsumH, Markestad T and Ueland PM. Cobalamin Status and Its Biochemical Markers Methylmalonic Acid and Homocysteine in Different Age Groups from 4 Days to 19 Years. Clin Chem 2003;49: 2067-2075.

9. Setola E, Monti LD, Gallucio E, Palloshi A, Fragasso G, Paroni $R$, et al. Insulin resistance and endothelial function are improved after folate and vitamin B12 therapy in patients with metabolic syndrome: relationship between homocysteine levels and hyperinsulinemia. Eur J Endocrinol 2004; 151: 483-489.

10. Bonora E, Kiechl S, Mayr A, Zoppini G, Targher G, Bonadonna R, et al. High-Normal HbA1c Is a Strong Predictor of Type 2 Diabetes in the General Population. Diabetes Care 2001;34: 1038-1040.

\section{Authors Contribution:} Concept of the article; GR - Concept of the article; ARS - Concept of the article.

Orcid ID:

Mr. C. Ananda Vayaravel: (1) http://orcid.org/0000-0002-2103-8417

Dr. R. Ramesh: (1) http://orcid.org/0000-0002-6939-4453

Dr. Shaik Anwar Hussain: (1) http://orcid.org/0000-0003-1411-7093

Dr. G.Radhika: http://orcid.org/0000-0002-5361-7595

Dr. A.R.Srinivasan: (1) https://orcid.org/0000.0001-5775-272X

Sources of support: None, Conflict of Interest: None declared.
}

CA - Concept of the article, manuscript preparation and critical revision of the manuscript; RR - Concept of the article and scrutinizing the manuscript; SAH - 